\title{
Assessment of Readability, Understandability, and Completeness of Pediatric Hospital Medicine Discharge Instructions
}

\author{
Ndidi I. Unaka, MD, MEd ${ }^{1 *}$, Angela Statile, MD, MEd ${ }^{1}$, Julianne Haney ${ }^{1}$, Andrew F. Beck, MD, MPH ${ }^{1,2}$, \\ Patrick W. Brady, MD, MSc ${ }^{1,3}$, Karen E. Jerardi, MD, MEd ${ }^{1}$
}

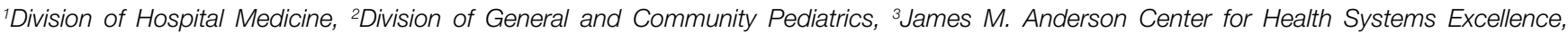
Cincinnati Children's Hospital Medical Center, Cincinnati, Ohio.
\end{abstract}

The average American adult reads at an 8th-grade level. Discharge instructions written above this level might increase the risk of adverse outcomes for children as they transition from hospital to home. We conducted a cross-sectional study at a large urban academic children's hospital to describe readability levels, understandability scores, and completeness of written instructions given to families at hospital discharge. Two hundred charts for patients discharged from the hospital medicine service were randomly selected for review. Written discharge instructions were extracted and scored for readability (Fry Readability Scale [FRS]), understandability (Patient Education Materials Assessment Tool [PEMAT]), and completeness (5 criteria determined by consensus). Descriptive statistics enumerated the distribution of readability, understandability, and completeness of written discharge instructions. Of the patients included in the study,
$51 \%$ were publicly insured. Median age was 3.1 years, and median length of stay was 2.0 days. The median readability score corresponded to a 10th-grade reading level (interquartile range, 8-12; range, 1-13). Median PEMAT score was 73\% (interquartile range, $64 \%-82 \%$; range, $45 \%-100 \%$ ); $36 \%$ of instructions scored below $70 \%$, correlating with suboptimal understandability. The diagnosis was described in only $33 \%$ of the instructions. Although explicit warning signs were listed in most instructions, $38 \%$ of the instructions did not include information on the person to contact if warning signs developed. Overall, the readability, understandability, and completeness of discharge instructions were subpar. Efforts to improve the content of discharge instructions may promote safe and effective transitions home. Journal of Hospital Medicine 2017;12:98-101. (C) 2017 Society of Hospital Medicine
The average American adult reads at an 8th-grade level. ${ }^{1}$ Limited general literacy can affect health literacy, which is defined as the "degree to which individuals have the capacity to obtain, process and understand basic health information and services needed to make appropriate health decisions."”,3 Adults with limited health literacy are at risk for poorer outcomes, including overuse of the emergency department and lower adherence to preventive care recommendations. ${ }^{4}$

Children transitioning from hospital to home depend on their adult caregivers (and their caregivers' health literacy) to carry out discharge instructions. During the immediate postdischarge period, complex care needs can involve new or changed medications, follow-up instructions, home care instructions, and suggestions regarding when and why to seek additional care.

The discharge education provided to patients in the hospital is often subpar because of lack of standardization and divided responsibility among providers. ${ }^{5}$ Communication of vital information to patients with low health literacy has been noted to be particularly poor, ${ }^{6}$ as many patient education materials are written at 10th-, 11th-, and 12th-grade

\footnotetext{
*Address for correspondence and reprint requests: Ndidi I. Unaka, MD, MEd, Division of Hospital Medicine, Cincinnati Children's Hospital Medical Center, 3333 Burnet Ave, ML 5018, Cincinnati, OH 45229; Telephone: 513-6368354; Fax: 513-636-7905; E-mail: ndidi.unaka@cchmc.org
}

Received: May 19, 2016; Revised: August 15, 2016; Accepted: August 31, 2016 2017 Society of Hospital Medicine DOI 10.12788/jhm.2688 reading levels. ${ }^{4}$ Evidence supports providing materials written at 6th-grade level or lower to increase comprehension. ${ }^{7}$ Several studies have evaluated the quality and readability of discharge instructions for hospitalized adults, ${ }^{8,9}$ and one study found a link between poorly written instructions for adult patients and readmission risk. ${ }^{10}$ Less is known about readability in pediatrics, in which education may be more important for families of children most commonly hospitalized for acute illness.

We conducted a study to describe readability levels, understandability scores, and completeness of written instructions given to families at hospital discharge.

\section{METHODS}

\section{Study Design and Setting}

In this study, we performed a cross-sectional review of discharge instructions within electronic health records at Cincinnati Children's Hospital Medical Center (CCHMC). The study was reviewed and approved by CCHMC's Institutional Review Board. Charts were randomly selected from all hospital medicine service discharges during two 3-month periods of high patient volume: January-March 2014 and January-March 2015.

CCHMC is a large urban academic referral center that is the sole provider of general, subspecialty, and critical pediatric inpatient care for a large geographical area. CCHMC, which has 600 beds, provides cares for many children who live in impoverished settings. Its hospital medicine service 
consists of 4 teams that care for approximately 7000 children hospitalized with general pediatric illnesses each year. Each team consists of 5 or 6 pediatric residents supervised by a hospital medicine attending.

Providers, most commonly pediatric interns, generate discharge instructions in electronic health records. In this nonautomated process, they use free-text or nonstandardized templates to create content. At discharge, instructions are printed as part of the postvisit summary, which includes updates on medications and scheduled follow-up appointments. Bedside nurses verbally review the instructions with families and provide printed copies for home use.

\section{Data Collection and Analysis}

A random sequence generator was used to select charts for review. Instructions written in a language other than English were excluded. Written discharge instructions and clinical information, including age, sex, primary diagnosis, insurance type, number of discharge medications, number of scheduled appointments at discharge, and hospital length of stay, were abstracted from electronic health records and anonymized before analysis. The primary outcomes assessed were discharge instruction readability, understandability, and completeness. Readability was calculated with Fry Readability Scale (FRS) scores, ${ }^{11}$ which range from 1 to 17 and correspond to reading levels (score $1=1$ st-grade reading level). Health literacy experts have used the FRS to assess readability in health care environments. ${ }^{12}$

Understandability was measured with the Patient Education Materials Assessment Tool (PEMAT), a validated scoring system provided by the Agency for Healthcare Research and Quality. ${ }^{13}$ The PEMAT measures the understandability of print materials on a scale ranging from $0 \%$ to $100 \%$. Higher scores indicate increased understandability, and scores under $70 \%$ indicate instructions are difficult to understand.

Although recent efforts have focused on the development of quality metrics for hospital-to-home transitions of pediatric patients, ${ }^{14}$ during our study there were no standard items to include in pediatric discharge instructions. Five criteria for completeness were determined by consensus of 3 pediatric hospital medicine faculty and were informed by qualitative results of work performed at our institution - work in which families noted challenges with information overload and a desire for pertinent and usable information that would enhance caregiver confidence and discharge preparedness. ${ }^{15}$ The criteria included statement of diagnosis, description of diagnosis, signs and symptoms indicative of the need for escalation of care (warning signs), the person caregivers should call if worried, and contact information for the primary care provider, subspecialist, and/or emergency department. Each set of discharge instructions was manually evaluated for completeness (presence of each individual component, number of components present, presence of all components). All charts were scored by the same investigator. A convenience sample of 20 charts was evaluated by a different investigator to ensure rating parameters were clear and classification was consistent
TABLE 1. Demographic Characteristics of Patients Whose Discharge Instructions Were Reviewed for Readability, Understandability, and Completeness $(\mathrm{n}=200)$

\begin{tabular}{lc}
\hline Characteristic & $\mathrm{N}(\%)$ \\
\hline Median (IQR) age, y & $3.1(2.2-8.9)$ \\
\hline Female & $101(51)$ \\
\hline Public insurance & $101(51)$ \\
\hline Most common discharge diagnoses & $56(28)$ \\
Bronchiolitis & $16(8)$ \\
Pneumonia & $11(5.5)$ \\
Asthma & $9(4.5)$ \\
Acute gastroenteritis & $8(4)$ \\
Brief resolved unexplained event & \\
\hline Hospital length of stay, d & $83(41)$ \\
1 & $84(42)$ \\
2 & $33(17)$ \\
$\geq 3$ & \\
\hline Number of discharge appointments & $110(55)$ \\
0 & $45(22.5)$ \\
1 & $45(22.5)$ \\
\hline 2 & $110(55)$ \\
\hline Number of discharge medications & $45(22.5)$ \\
0 & $79(39.5)$ \\
\hline NOTE: Abbreviation: IQR, interquartile range. & $47(23.5)$ \\
\hline & \\
\hline & \\
\hline & \\
\hline & \\
\hline &
\end{tabular}

(defined as perfect agreement). If the primary rater was undecided on a discharge instruction score, the secondary rater rated the instruction, and consensus was reached.

Means, medians, and ranges were calculated to enumerate the distribution of readability levels, understandability scores, and completeness of discharge instructions. Instructions were classified as readable if the FRS score was 6 or under, as understandable if the PEMAT score was under 70\%, and as complete if all 5 criteria were satisfied. Descriptive statistics were generated for all demographic and clinical variables.

\section{RESULTS}

Of the study period's 3819 discharges, 200 were randomly selected for review. Table 1 lists the demographic and clinical information of patients included in the analyses. Median FRS score was 10, indicating a 10th-grade reading level (interquartile range, $8-12$; range, 1-13) (Table 2). Only 14 (7\%) of 200 discharge instructions had a score of 6 or under. Median PEMAT understandability score was $73 \%$ (interquartile range, $64 \%-82 \%$ ), and $36 \%$ of instructions had a PEMAT score under $70 \%$. No instruction satisfied all 5 of the defined characteristics of complete discharge instructions (Table 2).

\section{DISCUSSION}

To our knowledge, this is the first study of the readability, understandability, and completeness of discharge instruc- 
TABLE 2. Descriptive Statistics Illustrating Readability, Understandability, and Completeness of Written Discharge Instructions

\begin{tabular}{lcc}
\hline Outcome & Calculated Value & Interpretation \\
\hline Readability & $10^{\mathrm{a}}(8-12)$ & 10th-grade reading level (8th-12th grade) \\
Median FRS score (1-17), (IQR) & $73 \%(64 \%-82 \%)$ & Score $<70 \%$ indicates text is difficult to understand \\
\hline Understandability & & \\
Median PEMAT score (0\%-100\%)(IQR) & $146(73 \%)$ & \\
\hline Completeness: number of instructions with: & $66(33 \%)$ & \\
Discharge diagnosis & $164(82 \%)$ \\
Description of diagnosis & $125(63 \%)$ & \\
Explicit warning signs & $23(12 \%)$ & $0(0 \%)$ \\
Information on person to contact with concerns &
\end{tabular}

aScore corresponds to grade level.

NOTE: Abbreviations: FRS, Fry Readability Scale; IQR, interquartile range; PEMAT, Patient Education Materials Assessment Tool.

tions in a pediatric population. We found that the majority of discharge instruction readability levels were 10 th grade or higher, that many instructions were difficult to understand, and that important information was missing from many instructions.

Discharge instruction readability levels were higher than the literacy level of many families in surrounding communities. The high school dropout rates in Cincinnati are staggering; they range from $22 \%$ to $64 \%$ in the 10 neighborhoods with the largest proportion of residents not completing high school. ${ }^{16}$ However, such findings are not unique to Cincinnati; low literacy is prevalent throughout the United States. Caregivers with limited literacy skills may struggle to navigate complex health systems, understand medical instructions and anticipatory guidance, perform child care and selfcare tasks, and understand issues related to consent, medical authorization, and risk communication. ${ }^{17}$

Although readability is important, other factors also correlate with comprehension and execution of discharge tasks. ${ }^{18}$ Information must be understandable, or presented in a way that makes sense and can inform appropriate action. In many cases in our study, instructions were incomplete, despite previous investigators' emphasizing caregivers' desire and need for written instructions that are complete, informative, and inclusive of clearly outlined contingency plans. ${ }^{15,19}$ In addition, families may differ in the level of support needed after discharge; standardizing elements and including families in the development of discharge instructions may improve communication. ${ }^{8}$

This study had several limitations. First, the discharge instructions randomly selected for review were all written during the winter months. As the census on the hospital medicine teams is particularly high during that time, authors with competing responsibilities may not have had enough time to write effective discharge instructions then. We selected the winter period in order to capture real-world instructions written during a busy clinical time, when pro- viders care for a high volume of patients. Second, caregiver health literacy and English-language proficiency were not assessed, and information regarding caregivers' race/ethnicity, educational attainment, and socioeconomic status was unavailable. Third, interrater agreement was not formally evaluated. Fourth, this was a single-center study with results that may not be generalizable.

In conclusion, discharge instructions for pediatric patients are often difficult to read and understand, and incomplete. Efforts to address these communication gaps-including educational initiatives for physician trainees focused on health literacy, and quality improvement work directed at standardization and creation of readable, understandable, and complete discharge instructions-are crucial in providing safe, high-value care. Researchers need to evaluate the relationship between discharge instruction quality and outcomes, including unplanned office visits, emergency department visits, and readmissions.

Disclosure: Nothing to report.

\section{References}

1. Kutner MA, Greenberg E, Jin Y, Paulsen C. The Health Literacy of America's Adults: Results From the 2003 National Assessment of Adult Literacy. Washington, DC: US Dept of Education, National Center for Education Statistics; 2006. NCES publication 2006483. https://nces.ed.gov/pubs2006/2006483.pdf. Published September 2006. Accessed December 21, 2016.

2. Ratzan SC, Parker RM. Introduction. In: Selden CR, Zorn M, Ratzan S, Parker RM, eds. National Library of Medicine Current Bibliographies in Medicine: Health Literacy. Bethesda, MD: US Dept of Health and Human Services, National Institutes of Health; 2000:v-vi. NLM publication CBM 2000-1. https://www.nlm.nih.gov/ archive//20061214/pubs/cbm/hliteracy.pdf. Published February 2000. Accessed December 21, 2016.

3. Arora VM, Schaninger C, D'Arcy M, et al. Improving inpatients' identification of their doctors: use of FACE cards. Jt Comm J Qual Patient Saf. 2009;35(12):613-619.

4. Berkman ND, Sheridan SL, Donahue KE, et al. Health literacy interventions and outcomes: an updated systematic review. Evid Rep Technol Assess (Full Rep). 2011;(199):1-941.

5. Ashbrook L, Mourad M, Sehgal N. Communicating discharge instructions to patients: a survey of nurse, intern, and hospitalist practices. J Hosp Med. 2013;8(1):36-41

6. Kripalani S, Jacobson TA, Mugalla IC, Cawthon CR, Niesner KJ, Vaccarino V. 
Health literacy and the quality of physician-patient communication during hospitalization. J Hosp Med. 2010;5(5):269-275.

7. Nielsen-Bohlman L, Panzer AM, Kindig DA, eds; Committee on Health Literacy, Board on Neuroscience and Behavioral Health, Institute of Medicine. Health Literacy: A Prescription to End Confusion. Washington, DC: National Academies Press; 2004.

8. Hahn-Goldberg S, Okrainec K, Huynh T, Zahr N, Abrams H. Co-creating patient-oriented discharge instructions with patients, caregivers, and healthcare providers. J Hosp Med. 2015;10(12):804-807.

9. Lauster CD, Gibson JM, DiNella JV, DiNardo M, Korytkowski MT, Donihi AC. Implementation of standardized instructions for insulin at hospital discharge. J Hosp Med. 2009;4(8):E41-E42.

10. Howard-Anderson J, Busuttil A, Lonowski S, Vangala S, Afsar-Manesh N. From discharge to readmission: understanding the process from the patient perspective. J Hosp Med. 2016;11(6):407-412.

11. Fry E. A readability formula that saves time. J Reading. 1968;11:513-516, 575-578.

12. D'Alessandro DM, Kingsley P, Johnson-West J. The readability of pediatric patient education materials on the World Wide Web. Arch Pediatr Adolesc Med. 2001;155(7):807-812.

13. Shoemaker SJ, Wolf MS, Brach C. The Patient Education Materials Assessment Too (PEMAT) and User's Guide: An Instrument to Assess the Understandability and Actionability of Print and Audiovisual Patient Education Materials. Rockville, MD: US Dept of
Health and Human Services, Agency for Healthcare Research and Quality; 2013. http://www.ahrq.gov/professionals/prevention-chronic-care/improve/self-mgmt/pemat/index.html. Published October 2013. Accessed November 27, 2013.

14. Leyenaar JK, Desai AD, Burkhart Q, et al. Quality measures to assess care transitions for hospitalized children. Pediatrics. 2016;138(2).

15. Solan LG, Beck AF, Brunswick SA, et al; H2O Study Group. The family perspective on hospital to home transitions: a qualitative study. Pediatrics. 2015;136(6):e1539-e1549.

16. Maloney M, Auffrey C. The Social Areas of Cincinnati: An Analysis of Social Needs: Patterns for Five Census Decades. 5th ed. Cincinnati, OH: University of Cincinnati School of Planning/United Way/University of Cincinnati Community Research Collaborative; 2013. http:/www.socialareasofcincinnati.org/files/FifthEdition/ SASBook.pdf. Published April 2013. Accessed December 21, 2016.

17. Rothman RL, Yin HS, Mulvaney S, Co JP, Homer C, Lannon C. Health literacy and quality: focus on chronic illness care and patient safety. Pediatrics. 2009;124(suppl 3):S315-S326.

18. Moon RY, Cheng TL, Patel KM, Baumhaft K, Scheidt PC. Parental literacy level and understanding of medical information. Pediatrics. 1998;102(2):e25.

19. Desai AD, Durkin LK, Jacob-Files EA, Mangione-Smith R. Caregiver perceptions of hospital to home transitions according to medical complexity: a qualitative study. Acad Pediatr. 2016;16(2):136-144. 